\title{
ORGANIZACIONES PRIVADAS DEL SECTOR SALUD DE MARACAIBO: ESTRATEGIAS FINANCIERAS Y VENTAJAS COMPETITIVAS
}

\author{
Carlos Borjas \\ Universidad del Zulia (Venezuela) \\ cborjas@gmail.com
}

\begin{abstract}
Resumen
La presente investigación analiza las estrategias financieras para el desarrollo de ventajas competitivas en las organizaciones privadas del sector salud de Maracaibo, Venezuela. Emplea para ello una metodología descriptiva que comprende el análisis documental y la aplicación de una encuesta, como instrumento de recolección de datos, dirigida a los administradores financieros de los centros de salud de la ciudad. En las estrategias financieras se observa la tendencia a conceder importancia a la generación de valor empresarial y el valor al accionista, así como una tipología dual de financiamiento, interno-externo, que inciden favorablemente en el desarrollo de ventajas competitivas y, por tanto, permiten enfrentar el dinamismo que impone el entorno.
\end{abstract}

Palabras clave: estrategias financieras, ventajas competitivas, sector salud, Maracaibo, Venezuela.

\begin{abstract}
The objective of the research was to analyze the financial strategies for developing competitive advantages in the private health sector organizations in Maracaibo, Venezuela, through a descriptive methodology that required documental analysis and the application of a survey as tool for data collection, to the financial managers of the health centres of the city. A trend was found in the financial strategies of giving importance to the generation of business value and shareholder value, as well as a dual type of internal-external funding that positively affect the development of competitive advantages that allow them to face the dynamism imposed by the context.
\end{abstract}

Key words: Financial strategies, competitive advantages, health sector, Maracaibo, Venezuela. 


\section{Introducción}

La globalización del sistema económico mundial, los avances tecnológicos, el desarrollo de las telecomunicaciones y de la informática, así como la creciente competencia en los mercados exigen, tanto a los gobiernos como a las empresas, la formulación y la implantación de estrategias para adaptarse exitosamente a un entorno cada vez más dinámico y cargado de incertidumbre.

En este orden de ideas, Thompson y Strickland (2004) refieren que las estrategias competitivas consisten en todos los movimientos y enfoques que ponen en práctica las empresas para atraer compradores, tolerar las presiones competitivas y mejorar su posición en el mercado. En términos más sencillos, consisten en tratar de desarmar a los rivales y obtener, a su vez, ventajas para ellas.

Al respecto, Luque (2001) señala que para formular estrategias competitivas, la organización debe conformar una gerencia estratégica que proporcione una visión amplia y a largo plazo de la situación de la empresa y establezca políticas dirigidas a la consecución de los objetivos planteados.

De la misma forma, esta gerencia debe conducir a la formulación, la ejecución y la evaluación de lineamientos en diversas áreas como mercadotecnia, recursos humanos, producción, investigación y desarrollo, y finanzas. Una de las aplicaciones más actualizadas se encuentra en materia financiera, cuya instrumentación práctica da origen al diseño de estrategias dirigidas a la recolección y el procesamiento de información externa e interna de la empresa, para establecer lineamientos orientados al costeo, el presupuesto, la elaboración de flujos de efectivo y la preparación de estados financieros proyectados. Así, el objetivo de la planeación estratégica es modelar el negocio a fin de generar utilidades satisfactorias.

A causa de la complejidad de las organizaciones, las distintas gerencias se ven en la necesidad de implementar una serie de estrategias financieras para obtener los recursos necesarios que les permitan el adecuado desarrollo de sus funciones. Por ello, los gerentes deben ser líderes capaces de identificar los aspectos que puedan afectar positiva o negativamente a la empresa y estar preparados para enfrentar cualquier situación que se presente. Deben tratar de alcanzar sus metas y, al mismo tiempo, mantener las ventajas competitivas que identifican a la organización.

Por otro lado, la situación económica actual de Venezuela y la intensa competencia, que exige cada vez mayor solidez financiera, hacen imperativa la necesidad de administrar eficientemente los recursos de las empresas con el fin de garantizar la permanencia de estas en el mercado. Esta realidad también afecta a las organizaciones privadas del sector salud de Maracaibo, donde opera una gran cantidad de servicios médicos privados dispuestos a cubrir la mayoría de las necesidades primarias del usuario-paciente y a brindarle, a él y a sus familiares, el mayor bienestar en términos de atención, comodidad y tranquilidad.

Por ser organizaciones tan complejas y estar al servicio de los distintos segmentos de usuarios de la ciudad que soliciten la prestación de servicios de salud, es trabajo de sus administradores gestionar la salud integral, entendida no solo como el intento de evitar enfermedades, sino como 
el correcto y armónico funcionamiento de sus organizaciones, lo que conducirá a un adecuado estado de bienestar físico, moral y social. Es ahí donde una etapa de la administración, la planificación financiera, entra en juego. Con el propósito de minimizar y eliminar las pérdidas deben formularse estrategias que permitan la planeación de las necesidades y el uso de los fondos para procurar los recursos suficientes y aplicarlos a los fines de rentabilidad del negocio, $y$ en aras de fomentar ventajas competitivas en el sector (Yépez, 2004).

De esta manera, surge la inquietud por conocer cómo son las estrategias financieras para el desarrollo de ventajas competitivas en las organizaciones privadas del sector salud de Maracaibo. El estudio comprende el análisis de los informes de gestión y financieros de este tipo de empresas y la aplicación de una encuesta, como instrumento de recolección de datos, a los administradores financieros de la totalidad de las organizaciones que prestan servicios de salud en la ciudad y que se encuentran debidamente registradas ante el Ministerio del Poder Popular para la Salud. El propósito es identificar los elementos del proceso de planificación, los objetivos y la tipología de las estrategias financieras y establecer cómo a partir de estas se desarrollan las ventajas competitivas aplicadas por las organizaciones privadas del sector salud en la ciudad. De los resultados del estudio deriva información valiosa que orientará las políticas y permitirá a las empresas tomar decisiones adecuadas y garantizar el retorno de su inversión.

En vista de la naturaleza descriptiva del estudio, la metodología empleada implicó la revisión de la literatura existente, la definición y la construcción de los instrumentos de recolección de datos y, finalmente, la aplicación de estos instrumentos. En primer lugar, se revisó la documentación disponible relativa a la planificación (estrategias formuladas), gestión y resultados (acciones ejecutadas) financieros de las organizaciones para el último ejercicio fiscal con el fin de analizar sus contenidos y comprender la situación actual y los condicionantes de esta. Seguidamente, se complementó la información con las opiniones de los administradores financieros a fin de recabar elementos adicionales que pudieran ampliar las perspectivas del análisis. Por último, la información empírica se confrontó con los aportes teóricos obtenidos de la revisión de la literatura para interpretar los resultados y garantizar el rigor metodológico indispensable, así como para contar con argumentos válidos que sustenten las consideraciones finales.

\section{Estrategias financieras en las organizaciones}

Según Diez y López (2001), la estrategia financiera en toda organización se define como la elección de los caminos por seguir para el logro de los objetivos financieros y, por ende, necesita ser organizada, presupuestada, supervisada y dirigida para que funcione en el sentido buscado. Al respecto, la estrategia financiera, como toda subestrategia, debe estar en consonancia con la estrategia general y tener en cuenta a los participantes internos y externos. Asimismo, debe centrarse en los aspectos financieros de las decisiones estratégicas, ligados al interés de los accionistas y los mercados financieros. La efectividad de la estrategia financiera se mide por su contribución al objetivo de la empresa, y la cuantificación de los resultados se mide igual que el objetivo de la empresa, esto es, en términos monetarios. 
Cabe destacar que las estrategias financieras incluyen dos tipos de subestrategias fundamentales: las estrategias de inversión, vinculadas con el establecimiento de pautas de selección de inversiones con diversificación del riesgo, y las estrategias de financiación, que buscan establecer la estructura idónea de las fuentes de recursos financieros.

Lo anterior refiere a la noción de planificación financiera, entendida por Bodie y Merton (2003) como el proceso dinámico que sigue un ciclo de trazar planes, instrumentarlos y revisarlos a la luz de los resultados, teniendo como punto de partida el plan estratégico de la compañía. Esta definición remite a la planificación financiera como el proceso por el cual la empresa trata de proyectar en términos monetarios el resultado futuro que desea alcanzar, intentando identificar los recursos que necesita para lograrlo.

Al respecto, Santandreu y Santandreu (2000) opinan que realizar una buena planificación financiera supone definir claramente los objetivos por alcanzarse a corto, mediano y largo plazo, establecer las acciones que se llevarán a cabo para conseguir dichos objetivos, y cuantificar los recursos humanos y económicos que tendrán que invertirse. Por ello, la planificación financiera, vista como un proceso, incluye una serie de elementos fundamentales que hacen de las finanzas una herramienta útil para el logro de los objetivos organizacionales: a) análisis del entorno, b) establecimiento de objetivos y metas y c) definición de los mecanismos de control y evaluación del desempeño.

Acorde con los planteamientos de Ortiz (2005), las estrategias financieras deben cimentarse en decisiones que contribuyan a la sincronización perfecta de los flujos monetarios, en forma tal que la integración de los montos recaudados y las disponibilidades iniciales de efectivo permitan el cumplimiento oportuno de los compromisos de deuda, como requisito que favorece el mantenimiento de buenas relaciones laborales, comerciales y financieras. Además, las estrategias deberán responder por el uso eficiente de los recursos, para evitar situaciones de saturación o ausencia, en función de lograr las metas de rentabilidad de la organización.

Según este precepto, a cada tipo de inversión le corresponde fuentes específicas de financiamiento de conformidad con el plazo previsto para recuperar los fondos invertidos. Las estrategias financieras abordan el objetivo crucial del destino apropiado de los fondos suministrados por el sistema financiero y los inversionistas. Por tanto, gracias a la consideración de aportes en función de la minimización de costos, el empleo efectivo de los recursos colocados a disposición de la gerencia y la generación de fondos vitales para el desempeño empresarial, las estrategias financieras auspician la recuperación de la inversión, por cuanto, contribuyen al alcance de resultados positivos en sus componentes: margen de utilidad y movilización o rotación de fondos, que pueden provenir tanto de fuentes externas como internas.

De acuerdo con Gitman (2000), se considera financiamiento interno a los fondos obtenidos de las operaciones propias de la organización: venta, operación o liquidación del inventario y capitalización de los dividendos, mientras que las fuentes externas son los fondos provenientes de personas o instituciones distintas de la organización. El financiamiento externo resulta evidente porque el patrimonio no puede satisfacer 
todas las necesidades de financiamiento de la organización, y en Venezuela el sistema bancario es el proveedor tradicional de estos fondos.

\section{Desarrollo de ventajas competitivas}

Asevera Porter (2006) que una organización está en posesión de ventajas competitivas sobre sus competidores si su rentabilidad está por encima de la rentabilidad media de las actividades del sector en el que se desempeña, y para ser realmente efectiva, una ventaja competitiva debe ser difícil de imitar, única, posible de mantener, netamente superior a la competencia y aplicable a variadas situaciones. Coinciden Hill y Jones (2005) al referir que una empresa tiene una ventaja competitiva sobre sus rivales cuando su rentabilidad es superior a la del promedio de todas las compañías de su industria, y esta ventaja competitiva es sostenida cuando es capaz de mantener el rendimiento superior al promedio durante varios años.

Para Thompson y Strickland (2004), las competencias distintivas son la base para las ventajas competitivas porque representan la experiencia y los conocimientos o las habilidades que los rivales no tienen y que no pueden igualar fácilmente. Por consiguiente, una competencia distintiva es un activo especialmente valioso que tiene el potencial de convertirse en el mecanismo principal para el éxito de una compañía, a menos que sea superado por los recursos más poderosos de los rivales. En el contexto financiero, afirma Porter (2006) que en la actualidad existen cinco fuerzas que determinan la consecuencia de rentabilidad a largo plazo de un mercado o de algún segmento de este: a) amenazas de entradas de nuevos competidores, $b$ ) rivalidades entre los competidores, c) poder de negociación de los proveedores, d) poder de negociación de los compradores y e) amenaza de ingreso de productos sustitutos. La idea es que la organización evalúe sus objetivos y recursos frente a estas cinco fuerzas.

En vista de lo anterior, para obtener una ventaja competitiva una empresa necesita seguir estrategias que se fundamenten en sus recursos y competencias existentes y que a la vez sean capaces de formular estrategias que conformen recursos y competencias adicionales. Asimismo, la fuente de una ventaja competitiva es la creación de valor superior; para crearlo, la organización debe reducir sus costos o diferenciar sus productos o ambas cosas al mismo tiempo.

En consecuencia, los cuatros bloques genéricos de construcción de la ventaja competitiva son: eficiencia, calidad, innovación y actitud de respuesta a los clientes. La eficiencia superior le permite a la empresa reducir sus costos; la calidad superior, fijar un precio más alto y reducir sus costos; y el servicio superior al cliente, también establecer un precio mayor. La innovación superior puede conducir a precios superiores, sobre todo en el caso de las innovaciones del producto o menores costos unitarios, especialmente en las innovaciones de procesos.

\section{Estrategias financieras y ventajas competitivas en organizaciones privadas del sector salud de Maracaibo}

Como en la presente investigación las estrategias financieras y el desarrollo de ventajas competitivas eran el objeto de estudio, se analizaron los informes de gestión y financieros y los resultados de la 
aplicación de la encuesta desde el punto de vista de la formulación de las estrategias financieras. Ello implicó identificar los siguientes conceptos operacionales sobre los cuales reposan los postulados desarrollados en las secciones anteriores: elementos del proceso de planificación, objetivos perseguidos, tipología y ventajas competitivas desarrolladas por las organizaciones privadas del sector salud.

Con relación a los elementos del proceso de planificación, se encontró que las organizaciones valoran los aportes del análisis situacional interno y externo en el desarrollo de sus actividades y en la gerencia de sus servicios. Coinciden con Serna (2000) en cuanto a la concepción del análisis FODA como una herramienta fundamental para el crecimiento y el desarrollo de la organización, pues permite visualizar las acciones futuras e incorporar el estudio de todos los agentes de su entorno y que afectan su sistema. Por lo anterior, las empresas, en su mayoría, han venido visualizando sus actividades futuras en función de los elementos que giran en torno a ella y que interfieren en su desempeño.

De igual forma, se advierte que un elevado número de las organizaciones analizadas mantienen objetivos y metas empíricas e invariables, pues no se orientan bajo lineamientos administrativos e indicadores de desempeño específicos, herramientas por medio de las cuales se puede evaluar el progreso de la organización. Ello va en contraposición a la opinión que Diez y López (2001), quienes afirman que los objetivos y las metas constituyen el núcleo central en el proceso decisorio, pues definen las acciones por desarrollar; asimismo, puede decirse que son los escenarios que alcanzará la organización en un tiempo determinado que ha sido estimado y para lo cual se le asignan recursos. No obstante, en la mayoría de las organizaciones se definen estrategias que representan el plan de acción que les permite alcanzar una posición de negocios atractiva, así como el desarrollo de ventajas competitivas sustentables en el tiempo, tal como lo plantean Thompson y Strickland (2004). Lo anterior, supone el manejo de planes de acción que coadyuvan en la obtención de ventajas competitivas.

En cuanto al establecimiento de políticas financieras, las organizaciones en su mayoría consideran dentro de su proceso de planificación y desarrollo de estrategias el hecho de establecer dichas políticas, lo cual resulta favorable, pues Santandreu y Santandreu (2000) afirman que al elaborar una política financiera se establecen reglas o guías que expresan los limites dentro de los cuales determinadas acciones deben ocurrir, en función de convertir objetivos financieros en realidades. No obstante, con respecto a la elaboración de planes financieros, se observó que el proceso de formulación de estos se sitúa en términos medios, pues el $25 \%$ de las organizaciones afirma elaborar los planes tomando en cuenta factores como el tiempo y la flexibilidad, mientras que otro $25 \%$ lo hace frecuentemente. Lo anterior, supone la presencia de obstáculos para el desarrollo de ventajas competitivas, en virtud de que el plan financiero constituye el documento base sobre el cual reposan y se fundamentan las estrategias generadoras de dichas ventajas, por lo que su elaboración debe ser una práctica institucionalizada en cada organización.

Al estudiar los objetivos de las estrategias financieras, se encontró que las organizaciones consideran la generación de valor tanto para ellas mismas como para los accionistas, pero no la cobertura de riesgos. Con frecuencia, la mayoría de las instituciones 
diseñan estrategias financieras en función de aquellos objetivos que giran en torno a maximizar el valor, considerando criterios como liquidez, costos ponderados, utilización de recursos y recuperación de fondos. A juicio de Ortiz (2005), al atender a la sumatoria de los criterios anteriormente mencionados, la aplicación de estrategias orientadas al logro del valor empresarial auspicia la recuperación de la inversión, por lo que contribuye al alcance de resultados positivos.

En lo referente a la generación de valor para el accionista, las organizaciones coinciden ampliamente en incorporar dicho aspecto al momento de diseñar sus estrategias financieras, para alcanzar beneficio mutuo. En este sentido, Gitman (2000) establece que la función de los administradores financieros es lograr los objetivos de los accionistas tomando en cuenta la maximización de las utilidades, las ganancias por acción y la protección del patrimonio, de hacerlo así alcanzarán los objetivos propios. No obstante, lo anterior difiere notablemente al considerar la cobertura de riesgos, pues el $44 \%$ de la población encuestada admite que casi nunca incluye dicho factor en el diseño de estrategias, lo que se opone a lo señalado por Ortiz (2005), quien indica la pertinencia de señalar la necesidad de que la gestión empresarial tome en consideración el riesgo comercial y económico presente en los proyectos para proteger a la empresa ante cualquier evento inesperado en el escenario financiero.

La ausencia de la cobertura de riesgos como objetivo perseguido por las estrategias deviene en una falla importante para la consecución de ventajas competitivas en el orden financiero, pues estas nacen de manera incompleta en las organizaciones de salud al no cubrir un elemento tan importante en las finanzas como la protec- ción ante la incertidumbre. A pesar de ello, el evidente interés por priorizar la formulación de estrategias que prioricen la liquidez, el eficiente empleo de los recursos y la recuperación de la inversión ha mostrado que la generación de valor como objetivo financiero exclusivo ha sido capaz de influir de manera positiva, si bien no idónea, en el desarrollo de ventajas competitivas para las organizaciones privadas del sector salud de la ciudad.

Para el estudio de la tipología de las estrategias financieras, se consideraron dos categorías: a) financiamiento interno, que incluyó cuentas por cobrar, inventarios y políticas de dividendo, $y b$ ) financiamiento externo, que comprendió emisión de nuevas acciones, préstamos bancarios, emisión de bonos, cuentas por pagar y papeles comerciales.

Sobre el financiamiento interno, se encontró que en la gran mayoría de las organizaciones casi siempre se consideran las cuentas por cobrar y los inventarios como las principales fuentes de este tipo de financiamiento. Este hallazgo concuerda con Weston y Brigham (2006), quienes afirman que emplear estos rubros como fuentes de financiamiento interno produce resultados satisfactorios al servir de amortiguadores para separar las actividades de compra, producción y ventas de la empresa, de modo que no todas tengan que llevarse a cabo al mismo ritmo, y porque así los eventos inesperados no pueden causar destrozos, pues la empresa mantiene la capacidad de responder al mercado. La política de dividendos, en cambio, no juega un rol preponderante en la formulación de las estrategias financieras.

Sobre el financiamiento externo, se observó que las organizaciones no suelen 
recurrir a nuevas acciones, bonos, cuentas por pagar y papeles comerciales como fuentes proveedoras de recursos financieros. Este hallazgo se encuentra en contraposición con Guajardo (2002), quien afirma que estos instrumentos suponen valiosas alternativas para el financiamiento de proyectos de inversión especiales y el desempeño de las operaciones. En cambio, los préstamos bancarios son el tipo de financiamiento externo al que recurre la casi totalidad de las organizaciones estudiadas, lo que a criterio de Chillida (2002) supone una excelente opción siempre y cuando las empresas establezcan relaciones continuas y positivas con los bancos a los cuales se dirigen las solicitudes de crédito, ya que de este modo es posible negociar tasas de interés y plazos de pago favorables.

Al abordar el desarrollo de ventajas competitivas, se encontró que aproximadamente el $44 \%$ de las organizaciones afirma que frecuentemente se desarrollan mecanismos de competencias distintivas como vía para alcanzar ventajas competitivas, mientras que el 19\% manifiesta que lo hacen casi siempre. Estas empresas realizan estudios periódicos sobre aspectos financieros del sector y manejan plataformas tecnológicas en sus actividades financieras, entre otras competencias que las hacen especialmente atractivas en comparación con las compañías rivales. Se trata de fortalezas competitivas que no se pueden igualar fácilmente.

En este sentido, y tal como lo afirman Thompson y Strickland (2004), una competencia distintiva es una base para la ventaja competitiva porque representa la experiencia y los conocimientos o las habilidades que los rivales no tienen y que no pueden igualar fácilmente. Lo anterior conduce a inferir que un mayor número de organizaciones del sector se orienta a establecer estrategias financieras vinculadas a fortalecer sus competencias distintivas en función de obtener ventajas competitivas sostenibles, como la eficiencia en el uso de los recursos escasos, la calidad en la prestación del servicio de salud (entendida por los usuarios no solo como la excelencia en el desempeño profesional del personal médico y de enfermería, sino también como la cualidad humana de la atención recibida y la genuina preocupación por la salud del paciente), la innovación en los procesos medulares del servicio (uso de tecnologías modernas y de métodos de diagnóstico y tratamiento de acuerdo con el avance de la medicina) y la actitud de respuesta a los clientes o usuarios, tal como se logró apreciar en los datos analizados.

De esta manera, la mayoría de las empresas estudiadas se basan en el desarrollo de sus fuerzas competitivas para emplearlas en el diseño de estrategias financieras y obtener así ventajas competitivas. Entre otros aspectos, ellas disponen de barreras de entradas hacia nuevos competidores y servicios, negocian con proveedores y clientes y toman en cuenta la competitividad del sector. Esto corresponde con lo señalado por Porter (2006), quien indica que las fuerzas competitivas advierten que ya no es posible esperar por la acción del competidor para decidir cómo se va a reaccionar; es necesario anticiparse al entorno para enfrentar cualquier eventualidad.

Finalmente, el análisis integral de los lineamientos previamente expuestos permite afirmar que, en las organizaciones privadas del sector salud en Maracaibo, la planificación estratégica está presente en las funciones de diagnóstico situacional, 
formulación de estrategias y políticas financieras, pero se encuentran debilidades en cuanto a la formulación de objetivos, metas y planes financieros. No obstante, se observa una tendencia en las estrategias financieras de conceder importancia a la generación de valor empresarial y valor al accionista, pero no a la cobertura de riesgos; todo esto a través de estrategias que asumen una tipología dual de financiamiento: a) interno, en el que prevalecen las cuentas por cobrar y el inventario, $y$ b) externo, en el que la principal fuente son los préstamos de instituciones financieras, que inciden en el desarrollo de ventajas competitivas con las cuales estas empresas pueden enfrentar, a pesar de las fallas, el dinamismo del contexto donde operan.

\section{Consideraciones finales}

El análisis de las organizaciones privadas del sector salud de Maracaibo, en cuanto a sus estrategias financieras y ventajas competitivas, permite observar un sector que de manera global se distingue por formular estrategias financieras que conducen al desarrollo de valiosas ventajas competitivas para enfrentar los retos que impone la economía contemporánea.

Estas organizaciones se orientan por esquemas de administración estratégica que priorizan el diagnóstico situacional y el diseño de lineamientos y persiguen, fundamentalmente, la maximización del valor para el accionista, así como el incremento del valor empresarial. Ello, tomando en cuenta la preocupación por obtener mayor liquidez y respuesta inmediata, a la vez que se consigue el beneficio mutuo por medio del empleo de una estructura de financiamiento que recurre en mayor medida a fuentes externas (instituciones bancarias), cuentas por cobrar e inventarios.

Esta caracterización de los elementos intervinientes en la formulación de estrategias financieras para el sector revela el establecimiento de ventajas competitivas, que favorecen en mayor medida la explotación de los activos y atributos importantes (experiencias, capital humano y reputación), así como la negociación con los principales clientes y proveedores. 


\section{Referencias bibliográficas}

BODIE, Zvi y MERTON, Robert. 2003. Finanzas. Ciudad de México: Pearson Educación.

CHILLIDA, Carmelo. 2002. Análisis e interpretación de balances: situación financiera a corto plazo (Tomo I). Caracas: Ediciones de la Biblioteca de la Universidad Central de Venezuela.

DIEZ, Luis y LÓPEZ, Joaquín. 2001. Dirección financiera: planificación, gestión y control. Madrid: Prentice Hall.

GUAJARDO, Gerardo. 2002. Contabilidad financiera. Ciudad de México: McGrawHill.

GITMAN, Lawrence. 2000. Principios de administración financiera. Ciudad de México: Harla.

HILL, Charles y JONES, Gareth. 2005. Administración estratégica: un enfoque integrado. Ciudad de México: McGraw-Hill.

LUQUE, Enrique. 2001. Introducción al estudio de la contabilidad. Ciudad de México: Continental.
ORTIZ, Alberto. 2005. Gerencia financiera: un enfoque estratégico. Ciudad de México: McGraw-Hill.

PORTER, Michael. 2006. La ventaja competitiva. Buenos Aires: McGraw-Hill.

SANTANDREU, Eliseu y SANTANDREU, Pol. 2000. Manual de finanzas. Barcelona: Gestión 2000.

SERNA, Humberto. 2000. Gerencia estratégica, planeación y gestión: teoría y metodología. Bogotá: Editorial 3R.

THOMPSON, Arthur y STRICKLAND, A. J. 2004. Administración estratégica. Ciudad de México: Pearson Educación.

WESTON, Fred y BRIGHAM, Eugene. 2006. Fundamentos de administración financiera. Ciudad de México: McGraw-Hill.

YÉPEZ, Mauricio. 2004. Finanzas en el sector privado. Bogotá: Norma. 